\title{
Modelling the kinetics of leachate remediation using microalgae
}

\author{
$\underline{\text { R.G. Richards }}^{\text {a }}$ and B. Mullins ${ }^{\text {b,c,d }}$ \\ ${ }^{a}$ Griffith Centre for Coastal Management (GCCM), Griffith University, Queensland \\ ${ }^{b}$ School of Public Health, Curtin University, Western Australia \\ ${ }^{c}$ Fluid Dynamics Research Group, Curtin University, Western Australia \\ ${ }^{a}$ Atmospheric Environment Research Centre (AERC), Griffith University, Queensland \\ Email: r.richards@griffith.edu.au
}

\begin{abstract}
The remediation of leachate from (municipal) landfills is becoming an increasing challenge for many government authorities. There is mounting interest in using bioremediation as a means of stripping these contaminants from the leachate and concentrating it within biological material, typically microalgae. Additionally, there is significant interest in the production of lipids from waste streams using microalgae. Landfill leachate typically hosts a suite of inorganic contaminants and therefore it is of great interest to evaluate the ability of different microalgae to (1) survive, (2) grow and (3) accumulate a range of heavy metals under field conditions. While this provides realism, it does however, require a firm understanding of how the interacting biology and chemistry of the microalgae and leachate constituents interact including the potential for feedback loops, delays and nonlinear functional relationships. To this end, we propose the use of a system dynamics modelling framework to develop a 'stock' (reservoirs) and 'flow' system dynamics model that explores the algae growth dynamics and the heavy metal adsorption kinetics simultaneously.
\end{abstract}

We have developed a model that mimics the temporal evolution of metal removal from a leachate into a biological mixture comprising of four common marine microalgae species- Nanochloropsis, Pavlova lutheri, Tetraselmis chuii and Chaetoceros muelleri. The growth dynamics of the microalgae species is modelled using four separate stocks that represent the concentration of each of the four species with inputs and outputs consisting of growth and mortality respectively. Growth is light-limited while both growth and mortality are assumed to be temperature dependent. Similarly, the five metals monitored in the leachate (iron, manganese, barium, cerium and lanthanum) are each represented by a stock. The uptake kinetics of the metals (removal from the leachate) are modelled using adsorption kinetics, taking into account that there are a finite amount of adsorption sites on the microalgae.

The model is primarily parameterised from data obtained through pilot studies using the four marine microalgal species. A photobioreactor employing light regimes, mixing and aeration were dosed with landfill leachate and simultaneously seeded with the four microalgal species and left for ten days. The leachate and the microalgae species were analysed for metal content at the beginning and end of the batch experiment. The use of the reactor and subsequent results enables the fundamental microalgae growth kinetics (growth and mortality) to be simultaneously fitted to the data. It is assumed that any potential effects of leachate toxicity on microalgae growth dynamics will be implicitly included in the mortality rate constants.

Overall, this system dynamics model provides a mechanism for understanding and predicting the bioremediating ability of different algae under realistic conditions.

Keywords: dynamic modelling, microalgae, leachate remediation, heavy metals, batch reactor 


\section{INTRODUCTION}

Landfills are a common means of solid waste disposal in Australia, for both municipal and industrial wastes. As waste in a landfill breaks down, it releases liquid (termed leachate), which is due to both rainwater percolating through the landfill, and the breakdown of (usually putrescible) materials in the landfill. Landfill leachate is typically captured in holding ponds and then evaporated (where possible), or treated and disposed of via various means. Landfill leachate is typically composed of organic matter, decomposition products, organic chemicals (solvents etc.) and dissolved metals. Landfill leachate treatment typically consists of conventional aerobic or anaerobic treatment methods (Sunil et al. 2011; Ana-Maria and Maria 2010; Augustino et al. 2010). While such treatment methods are capable of producing leachate of suitable quality for reuse or disposal, they are highly energy intensive, and do not allow the recovery of metals or lipid production from the wastewater.

It has been shown that many species of microalgae are suited to the processing of nutrient-rich wastewater sources, and commonly produce high lipid yields, which may be beneficial for a range of uses. In addition, microalgae are commonly able to bioaccumulate metals, which can allow efficient removal of metals from wastewater streams (Boke and Seda 2010). However, the treatment of (landfill) leachate using microalgae has received very little attention (Elena et al. 2010; Lin et al. 2007; Cheung et al. 1993). Cheung et al. (1993) examined the toxicity of landfill leachate on four freshwater microalgal species - two Chlorella sp., Scenedesmus sp. and Dunaliella sp. It was found that the leachate inhibited the growth of all algae species, however particularly the Chlorella sp., which normally produce the highest lipid yields of the species used. In order to understand systems that can reliably treat leachate using microalgae, it is important to develop models to allow system investigation and optimization. It is known that saline species of microalgae are generally more resilient and robust than freshwater species. In addition to the treatment and disposal of landfill leachate, the disposal of hypersaline water from desalination plants is also a significant problem. Desalination plants must frequently store hypersaline water if salinity in the disposal area becomes too high.

This work therefore investigates the potential of combining landfill leachate with hypersaline water, and simultaneously treating both waste streams using a mixed culture of marine microalgal species. Microalgae have the potential to simultaneously remove nutrients and metals from the leachate, and at the same time produce beneficial lipids.In order to accurately quantify the dynamics of such systems, it is essential to model the metals uptake from leachate, and the impact this has on growth rates and mortality. This paper will focus on the use of systems modelling techniques to explore the dynamics of such systems.

\section{MATERIALS AND METHODS}

\subsection{Model Description}

A stock and flow model (Figure 1) was developed using the software platform Vensim (http://www.vensim.com). Vensim is a computer software platform that enables the construction of dynamic models using 'stocks' and 'flows' to represent reservoirs (state variables e.g. algal concentration) and rates (e.g. mortality rate of algae) respectively. The biological component of the model represents the coupled dynamics of the four algal species $\left(\mathrm{ALG}_{i}\right.$, where $i$ subscript can take a value of 1, 2, 3 or 4 and indicates which of the four algal species is being modelled using equation 1) a pool of detritus and dissolved inorganic nutrients (NPD model). The growth dynamics of the algae is controlled by the multiplicative effects of temperature (fT), light (fL) and nutrients (fN). fT is modelled using the Arrhenius equation (Bowie et al. 1985), fL using Steele's equation (Steele 1962) and fN using a Michaelis-Menton function. Dead algae is assumed here to contribute directly to the pool of (organic) detritus located in the laboratory systems. Algal mortality is modelled using a temperature-referenced rate constant (mort $20, i-$ where $i$ reflects that the mortality rate is algal species specific with $\mathrm{i}=1,2,3$ or 4 ) adjusted for the effects of temperature using fT. The nitrogen (model currency) continues to be cycled via remineralisation of the detritus into dissolved inorganic nutrients (DIN) using a temperature referenced mineralisation rate constant $\left(\min _{20}\right)$ that is temperature adjusted. Cycling of $\mathrm{N}$ is completed through uptake of DIN by the algae. The state equations for algae, detritus and dissolved inorganic nitrogen are:

$$
\begin{aligned}
& \frac{\mathrm{d}\left[\mathrm{ALG}_{\mathrm{i}}\right]}{\mathrm{dt}}=\left(\mathrm{fL} \times \mathrm{fN} \times \mu_{\mathrm{MAX}}-\operatorname{mort}_{20}\right) \times \mathrm{ALG}_{\mathrm{i}} \times \mathrm{fT} \\
& \frac{\mathrm{d}[\mathrm{DET}]}{\mathrm{dt}}=\sum\left(\operatorname{mort}_{20, \mathrm{i}} \times \mathrm{fT} \times \mathrm{ALG}_{\mathrm{i}}\right)-\min _{20} \times \mathrm{fT} \times \mathrm{DET}
\end{aligned}
$$


Richards and Mullins, Modelling the kinetics of leachate remediation using microalgae

$$
\frac{\mathrm{d}[\mathrm{DIN}]}{\mathrm{dt}}=\min _{20} \times \mathrm{fT} \times \mathrm{DET}-\sum\left(\mathrm{ALG}_{\mathrm{i}} \times \mathrm{fL} \times \mathrm{fN} \times \mathrm{fT} \times \mu_{\mathrm{MAX}}\right)
$$

Each metal was represented in the model as a 'free' (non-adsorbed) concentration along with the respective concentrations adsorbed to each of the four algal species requiring the use of 25 state variables. Algalspecific adsorption rates (adjusted for temperature effects) were specified through calibration for each of the metals.

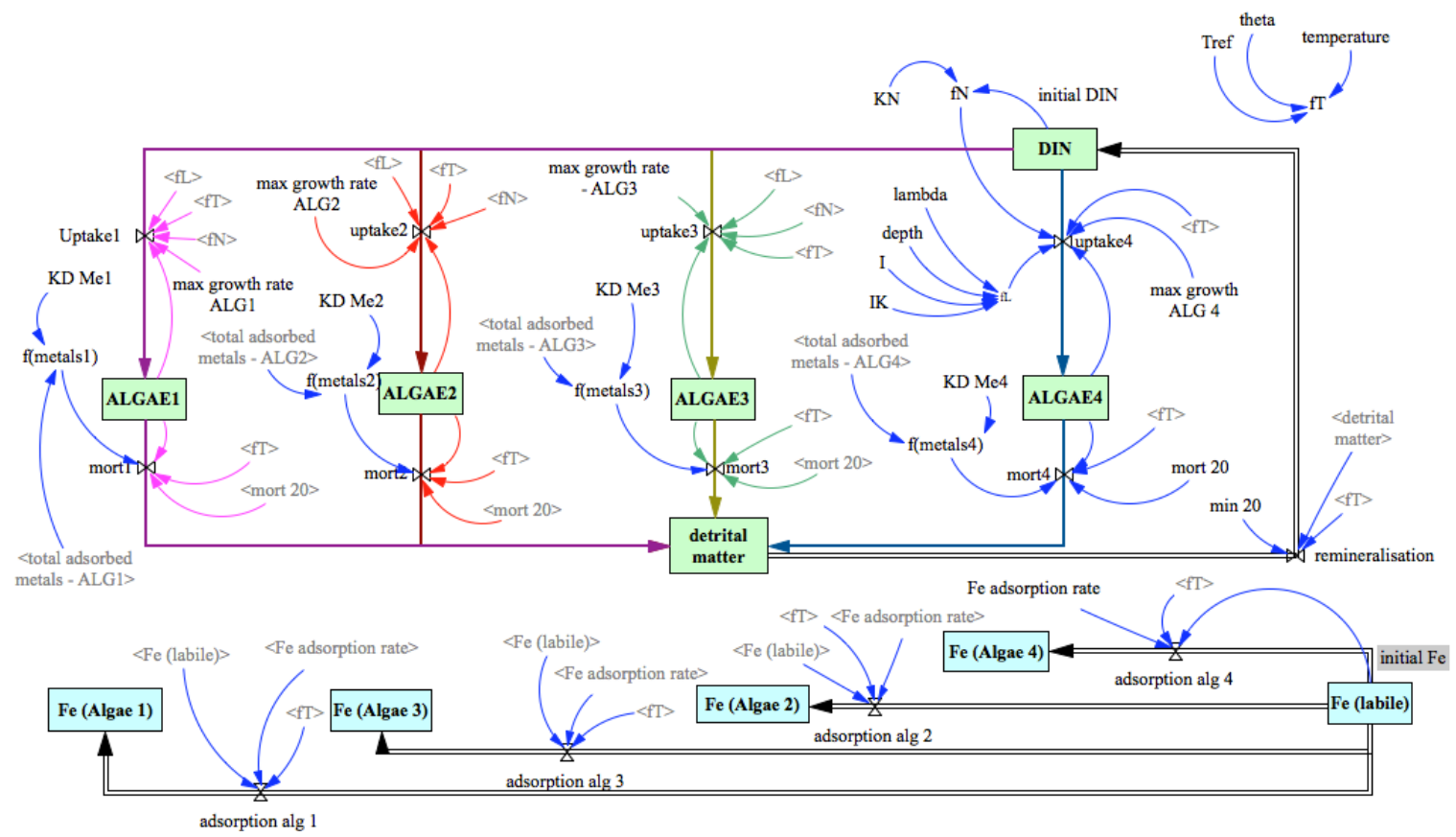

Figure 1. Stock and flow model of a single photobioreactor - metal kinetics only shown for iron (Fe).

The toxicity of metals on algal growth is related here to the total concentration of the five metals adsorbed to each algal species rather than individual effects because there is not metal-algae specific data to enable this to be achieved. The potential combined toxic effect of metals on algal growth was modelled using a MichaelisMenton function to adjust the background generic algal mortality rate that is specified for all algal species (see Table 1). A half-saturation constant is used to relate the adsorbed metal concentration to mort ${ }_{20}$. Key assumptions in the model include the assumption that there are unlimited metal binding sites for each algal species and the algal growth is not nutrient limited for the experimental period (i.e. algal growth is limited only by metal toxicity).

Table 1. Model Parameters

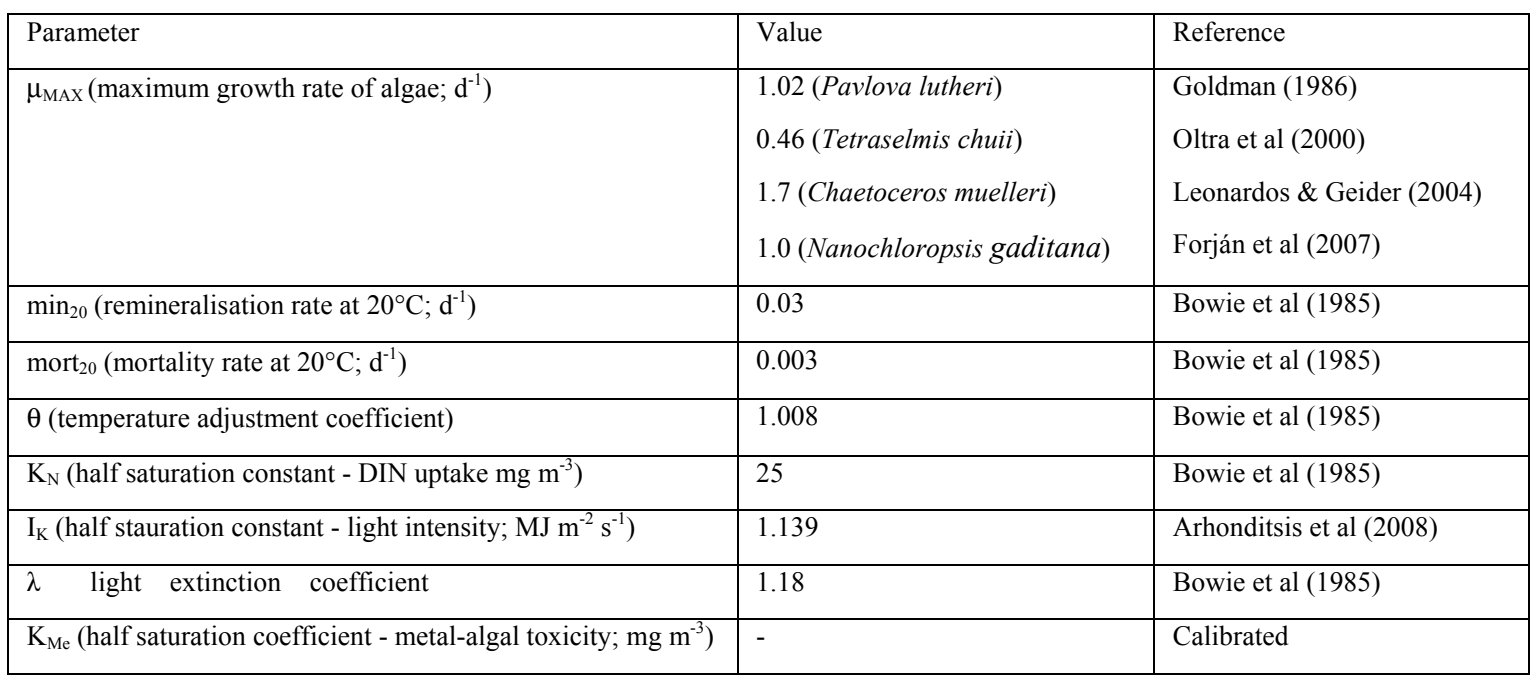




\subsection{Experimental set-up}

Typical municipal leachate samples were obtained from the leachate holding ponds of an operating landfill. The leachate was allowed to settle to remove any solid/particulate matter, and the supernatant used for the experiments. A full characterization of Biological Oxygen Demand (BOD), Chemical Oxygen Demand (COD), Suspended solids, and metals (via Inductively Coupled Plasma - Mass Spectrometry) was conducted on the raw leachate. A cylindrical photo-bioreactor was utilised. The operating volume of the reactor was 2.5 1. Three 5,000K (natural white) lamps (NEC, China) were fitted to the photobioreactor. The lamps were illuminated for 12 hours in each $24 \mathrm{hr}$ period, using a timer. The bioreactors were covered with an external layer of reflective material to improve overall light intensity and uniformity. The light intensity profile was measured and proven to be sufficiently uniform. Air was injected into the bottom of each reactor to ensure complete mixing and provide a suitable growth environment. The air flow rates of the reactor $(2.5 \mathrm{~L})$ was $1250 \mathrm{ml}$ $\min ^{-1}$.

Four species of the marine microalgae, Nanochloropsis, Pavlova lutheri, Tetraselmis chuii and Chetoceros muelleri, were used in the experiments. A $50 \mathrm{ml}$ solution of each species was added to the reactor, following the addition

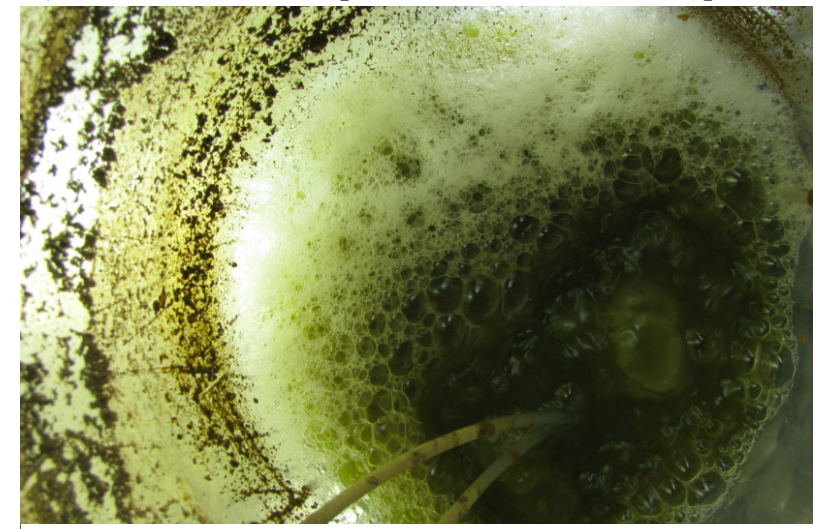

Figure 2. Image of the Reactor during operation, showing oxygen and carbon dioxide lines. of the leachate and an artificial sea-salt mixture (to simulate the mixture of leachate and hypersaline desalination water). An artificial sea-salt mix (Aquasonic) was added at a rate of $75 \mathrm{~g}$ synthetic sea salt to the Bioreactor; this simulated the addition of hypersaline water from the desalination process. The temperatures in the reactors was measured as $34.6 \pm 0.2^{\circ} \mathrm{C}$. A partial cover was used on the reactor to minimise evaporation.

\section{RESULTS}

The initial and final concentrations of the four algal species and the five metals are summarised in Table 2. All algal species increased their concentration by the end of the experimental period. All metal concentrations were observed to decrease over the experimental period. In particular, Fe decreased by $95 \%$ in the reactor.

Table 2. Initial and final conditions for the bioreactor

\begin{tabular}{|l|l|l|}
\hline \multirow{2}{*}{ Constituent } & Reactor \\
\cline { 2 - 3 } & Conc start & Conc end \\
\hline Pavlova lutheri (cells/ml) & $3 \times 10^{5}$ & $1.8 \times 10^{6}$ \\
\hline Tetraselmis chuii (cells/ml) & $3 \times 10^{5}$ & $6 \times 10^{5}$ \\
\hline Chaetoceros muelleri (cells/ml) & $3 \times 10^{5}$ & $4.8 \times 10^{6}$ \\
\hline Nanochloropsis gaditana (cells/ml) & $4 \times 10^{5}$ & $4.8 \times 10^{6}$ \\
\hline Iron (Fe) - ppb & 15370 & 683 \\
\hline Manganese (Mn) - ppb & 270 & 192 \\
\hline Barium (Ba) - ppb & 175 & 89 \\
\hline Cerium (Ce) - ppb & 7 & 0 \\
\hline Lanthanum (La) - ppb & 3 & 0 \\
\hline
\end{tabular}


The systems model was successfully fitted to the experimental data by minimising the least squares error between measurement and prediction. Note that for the aqueous concentrations of Ce and La which were recorded as zero concentrations at the end of the experiment, no data was available on how quickly (within the experimental timeframe) these metals were removed from the aqueous solution. For the purpose of this modelling exercise, it was assumed that the end concentration for both Ce and La were $0.05 \mu \mathrm{g}{ }^{-1}$ (values which represent the limits of detection), which then allowed the adsorption constants for these two metals to be calibrated.

Unsurprisingly, the model was found to be sensitive to the somewhat arbitrary end concentrations specified for Ce and La. This is an artefact of not having sufficient data to 'track' the decay of these two metals (basically we only have single data points from the data because concentrations are zero by the end of the experimental program). Suffice to say that the adsorption constants estimated for $\mathrm{Ce}$ and La are not particularly informative without specific knowledge on when these metal concentrations decreased to zero.

The model predictions for Fe and $\mathrm{Mn}$ concentrations in the reactor are presented in Figure 3. The aqueous concentration of $\mathrm{Fe}$ and $\mathrm{Mn}$ (and also for $\mathrm{Ba}, \mathrm{Ce}$ and $\mathrm{La}$ ) decreases exponentially as these respective metals are drawn from the water column and adsorbed to the four algal species. For Fe (Figure 3), metal adsorption was predicted in the model to be dominated by C. muelleri while for Mn (Figure 3), Ba and Ce the metal adsorption was more evenly distributed amongst the four algal species. These observations were consistent for both reactor environments.
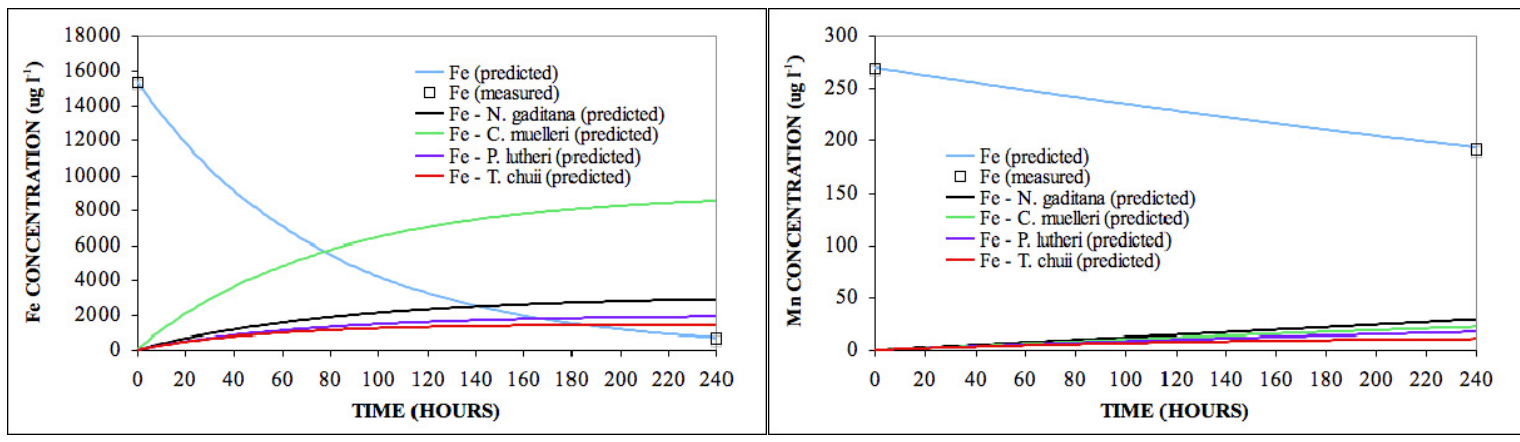

Figure 3. Speciation calculations for Fe (left panel) and Mn (right panel).

Model predictions on the algal growth dynamics in the reactor are presented in Figure 4. These are characterised by exponential growth of each species over the 10 day experimental period. Strongest growth (observed and predicted) was observed for N. gaditana and C. muelleri with both species reaching a concentration of ca. $900 \mathrm{mg} \mathrm{l}^{-1}$ after 10 days. Notably lower concentrations were observed for P. lutheri (ca. $300 \mathrm{mg} \mathrm{l}^{-1}$ ) and T. chuii (ca. $100 \mathrm{mg} \mathrm{l}^{-1}$ ).

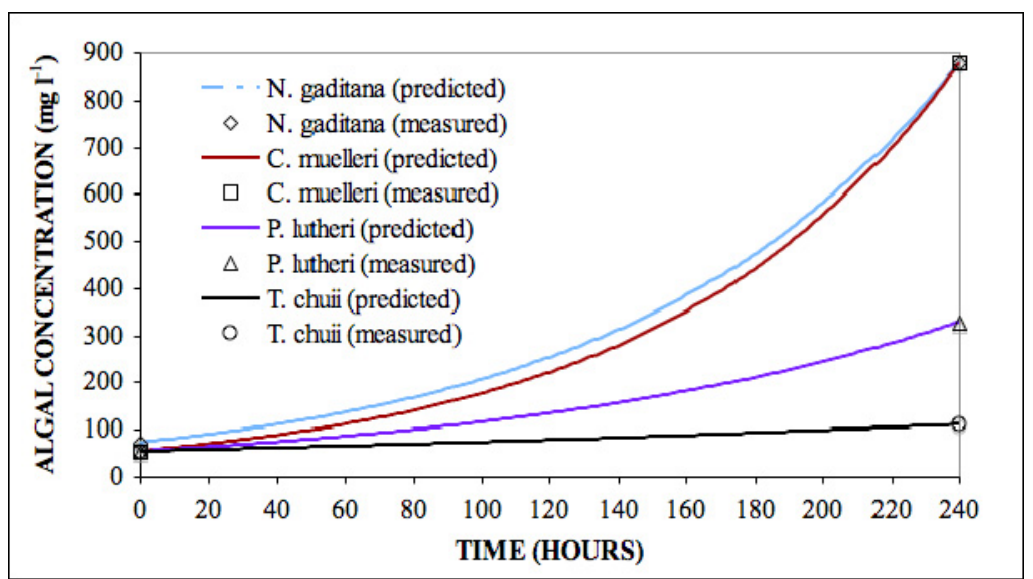

Figure 4. Predicted and measured concentrations of algae in the Reactor over the experimental period (10 days). 


\section{DISCUSSION AND CONCLUSIONS}

We present here a systems model that has been developed to explore the combined dynamics of algal growth, metal adsorption and metal toxicity. Overall, the method proposed appears a viable means of simultaneously treating both landfill leachate and disposing of hypersaline desalination water, provided the algal separation can be conducted efficiently. The algae generally showed excellent uptake of the metals. A key point of interest is that Tetraselmis sp. are known to dominate mixed culture systems, however this was not observed here. Rather, T. chuii was observed to exhibit the lowest concentration out of the four algal species. It is therefore evident that Tetraselmis sp. proved less resilient to the metals concentrations than the other species.

The systems dynamics modelling approach provides a means for exploring algal growth dynamics of a multispecies mixture and their ability to adsorb a suite of metals whilst also considering the potential effects of metal toxicity on algal growth. Understanding the 'cross' dynamics of metal adsorption and the accompanying toxic effect on algal growth dynamics are important considerations here.

By using basic algal growth functions (nutrient uptake, mortality) and coupling three compartments (algae, detritus and dissolved inorganic nutrients), the biological component of the model was able to 'match' the start and end concentrations of each of the four algal species with good accuracy. The absence of data across the experimental period entails that the functional form of the growth curves for the microalgae species is not verified here. However, exponential growth curves, which were observed for all species except T. chuii, are commonly observed in microalgae species under non-limiting or weakly limited conditions (e.g. Leonardos and Geider 2004).

The simplicity of the model and the relative dearth of parameterising data entailed that the model has major simplifications. The use of aggregate term to represent toxic effect of adsorbed metals on the different algal species was used because of the absence of metal-algal concentrations for each of the metals. This approach does not allow the toxicities of the individual metals (or different combinations of the different metals) on algal dynamics to be explored. Furthermore, the effect of metal toxicity will likely impact on both growth rates and mortality rates - we chose to focus on the effect on mortality rate only. We did, however, select to explicitly model the effect of temperature on growth and mortality rates using a standard adjustment algorithm (Arrhenius equation) as it is known to be a major influence on algal growth dynamics (e.g. Oltra et al. 2000). We used a generic mortality rate that was applied for all four algal species as these have been shown to be similar across algal species (Bowie et al. 1985).

From a decision-making and optimisation contexts, such systems dynamics modelling can provide an insight into the efficacy of leachate management in terms of maximising the available resources (or minimising the use of resources used). This is a practical challenge for decision makers when confronted with leachates that comprise of variable (dynamic) mixed contaminants that can affect the ability of the microalgae species extracting the contaminants. However, it is also important to highlight that numerical models are ultrasimplifications of reality and therefore what they can deliver can always by improved.

Overall, this model will be beneficial in selecting the most suitable algal species and optimizing the conditions at which to treat the leachate.

\section{ACKNOWLEDGMENTS}

The authors would like to acknowledge research funding from the (Perth) Eastern Metropolitan Regional Council. The authors would also like to acknowledge the assistance of Mr Simon Longbottom and Mr Xiao Hua.

\section{REFERENCES}

Agustiono, K.T., Waihung, L., Chan G., et al., (2010). Biological processes for treatment of landfill leachate. Journal of Environmental Monitoring. 12(11). 2032-2047.

Ana-Maria, S. and Maria, G., (2010). Municipal solid waste landfilling and treatment of resulting liquid effluents. Environmental Engineering and Management Journal. 9(7), 993-1019.

Boke, O.H. and Seda, T.Z., (2010). Assessment of various parameters of metal biology in marine microalgae Phaeodactylum Tricornutum and Dunaliella Tertiolecta. Fresenius Environmental Bulletin. 19(12A). 29812986.

Bowie, G.L., Mills, W.B., Porcella, D.B., Campbell, C.L., Pagenkopf, J.R., Rupp, G.L., Johnson, K.M., Chan, P.W.H., Gherini, S.A., Chamberlain, C.E. (1985). Rates, constants, and kinetics formulations in 
surface water quality modeling (Second Edition). Environmental Research Laboratory Office of Research and Development U.S. Environmental Protection Agency.

Cheung, K.C., Chu, L.M. and Wong M.H., (1993). Toxic effect of landfill leachate on microalgae. Water Air and Soil Pollution. 69(3-4). 337-349.

Elena, L., Vishwanath, P. and Elin, G., (2010). Efficient Purification of Heavy-Metal-Contaminated Water by Microalgae-Activated Pine Bark. Water Air and Soil Pollution. 210(1-4). 493-500.

Forján, E., Garbayo, I., Casal, C., Vílchez C. (2007). Enhancement of carotenoid production in Nannochloropsis by phosphate and sulphur limitation. Communicating Current Research and Educational Topics and Trends in Applied Microbiology A. Méndez-Vilas (Ed.)

Goldman, J.C., (1986). On phytoplankton growth rates and particulate C:N:P ratios at low light. Limnology and Oceanography. 31, 1358-1363.

Leonardos, N. and Geider, R.J. (2004). Effects of nitrate:phosphate supply ratio and irradiance on the C:N:P stoichiometry of Chaetoceros muelleri. Eur.J. Phycol. 39, 173-180.

Lin L., Chan G.Y.S., Jiang B.L., et al., (2007).Use of ammoniacal nitrogen tolerant microalgae in landfill leachate treatment. Waste Management. 27(10). 1376-1382.

Oltra, R., Todolí, R., Bosque, T., Lubián, L.M., Navarro, J.C. (2000). Life history and fatty acid composition of the marine rotifer Synchaeta cecilia valentina fed different algae. Marine Ecology Progress Series 193, $125-133$.

Steele, J.H. (1962). Environment control of photosynthesis in the sea. Limnology and Oceanography 7, 137150 .

Sunil, K., Chart, C. and Ackmez, M., (2011). Bioreactor landfill technology in municipal solid waste treatment: An overview. Critical Reviews in Biotechnology. 31(1), 77-97. 